\title{
Structure and Lateral Electron Hopping in Osmium-tris-4,7-diphenylphenanthroline Perchlorate Monolayers at the Air/Water Interface
}

\author{
Won-Yong Lee and Marcin Majda* \\ Department of Chemistry, University of California at Berkeley, Berkeley, California 94720-1460
}

Gerald Brezesinski

Max-Planck-Institute für Kolloid- und Grenzflächenforschung, Rudower Chaussee 5, D-12489 Berlin, Germany

\author{
Michael Wittek and Dietmar Möbius \\ Max-Planck-Institute für Biophysikalische Chemie, Postfach 2841, D-37018 Göttingen, Germany
}

Received: March 26, 1999; In Final Form: June 14, 1999

\begin{abstract}
Osmium tris-diphenylphenanthroline perchlorate $\left(\mathrm{Os}(\mathrm{DPP})_{3}\right)$ can be spread at the air/water interface where it forms solid monolayer films. Brewster angle microscopy revealed that these films consist of irregular ca. 100 to $1000 \mu \mathrm{m}$ diameter 2D aggregates which coalesce upon compression to form continuous films. Grazing incidence X-ray diffraction data showed that the structure of the aggregates is independent of the degree of monolayer compression and features a 2D lattice of hexagonally close-packed Os(DPP $)_{3}$ centers with Os-Os distances of $12.57 \AA$. The latter is 4 to $24 \%$ shorter than the $\mathrm{Ru}-\mathrm{Ru}$ distances found in the $3 \mathrm{D}$ monoclinic crystal of an isostructural Ru (DPP) 3 . Two dimensional electrochemical measurements carried out with line microelectrodes at the air/water interface were used to study kinetics of the lateral electron transport in these Langmuir monolayers. Electron transport involves electron hopping on the 2D lattice of the osmium sites where the individual electron transfer steps between $\mathrm{Os}^{\mathrm{II}}(\mathrm{DPP})_{3}$ and $\mathrm{Os}^{\mathrm{III}}(\mathrm{DPP})_{3}$ take place with the rate constant $k_{1}=4.7 \times 10^{8} \mathrm{~s}^{-1}$. Percolation theory was used to account for the observed increase of the electron hopping rates during monolayer compression on the water surface resulting in an increase of the extent of connectivity and thus electroactivity of the initially formed $2 \mathrm{D} \mathrm{Os}(\mathrm{DPP})_{3}$ aggregates. Percolation theory also accounts well for the dependence of the electron hopping rates on the composition of fully compressed Os$(\mathrm{DPP})_{3} / \mathrm{Ru}(\mathrm{DPP})_{3}$ monolayers in which the ruthenium species were used to homogeneously dilute the Os$(\mathrm{DPP})_{3}$ sites. In contrast, in Os(DPP $)_{3} /$ octadecanol monolayers, macroscopic self-segregation of the two components was inferred from a larger positive shift of the apparent percolation threshold in the lateral electron hopping.
\end{abstract}

\section{Introduction}

The mechanism and kinetics of electron transport processes in multimolecular systems have been subjects of intense and diverse investigations. Interest in this broad subject stems to a large extent from its relevance to biological electron transport processes. Investigations of electron transport concerned numerous supramolecular chemical systems created to model and reproduce functions of natural photosynthesis. ${ }^{1-5}$ Much research in this area has been concerned with the kinetics of long-range electron tunneling. ${ }^{6-16}$ Electron transport and related phenomena have also been a focus of substantial electrochemical investigations owing to the significance of these processes in electrocatalysis, in photoelectrochemical, electrochromic, and molecular electronic devices, and in electrochemical sensors. ${ }^{17-20}$ Efficient electron transport over micrometer distances in multimolecular, microstructural media encountered in these systems is of crucial importance to their efficiency.

Electrochemical investigations of charge transport addressed a variety of multimolecular thin film systems deposited on the electrode surface where electron propagation proceeds in and

*Electronic mail address: majda@socrates.berkeley.edu. involves the $3 \mathrm{D}$ space of a film matrix. ${ }^{20,21}$ In contrast, this report presents electrochemical investigations of the lateral electron transport kinetics in solid 2D monolayers of redox species in Langmuir monolayers at the air/water interface. Our long-term goal in this area is to use Langmuir monolayer techniques and 2D electrochemical methods to create systems of controlled structure and to investigate the dependence of the electron transfer kinetics on structural parameters such as intermolecular distance and relative orientation of the donoracceptor centers and the chemical nature of the intervening medium.

The monolayer consists of osmium tris-4,7-diphenyl-1,10phenanthroline perchlorate $\left(\mathrm{Os}(\mathrm{DPP})_{3}\right)$, a water insoluble salt spread at the air/water interface. ${ }^{22}$ We used Brewster angle microscopy 23 (BAM) to characterize monolayer morphology during compressions. Formation of small (ca. 100 to $1000 \mu \mathrm{m}$ diameter) 2D solid aggregates which can be compressed to form continuous films is apparent. The structures of these aggregates and the fully compressed films were determined using grazing incidence X-ray diffraction (GIXD). ${ }^{24,25}$ Subsequent electrochemical characterization of the electron transport kinetics involved "line" microelectrodes designed to function in the plane 
of the air/water interface where they can address electrochemically redox species forming monolayer films. ${ }^{26-28}$ Combination of these techniques allowed us to show that diffusive electron transport involves electron hopping between neighboring osmium sites with a unimolecular rate constant of $k_{1}=4.7 \times 10^{8}$ $\mathrm{s}^{-1}$. The electron hopping obeys percolation theory during monolayer compression when increasing connectivity between the individual aggregates and a line microelectrode leads to a gradual increase of the extent of electroactivity of the monolayer. Percolation theory is also obeyed when electron hopping kinetics is measured in fully compressed, mixed $\mathrm{Os}(\mathrm{DPP})_{3} / \mathrm{Ru}(\mathrm{DPP})_{3}$ monolayers in which $\mathrm{Ru}(\mathrm{DPP})_{3}$ is used as a homogeneous diluent of the osmium sites.

\section{Experimental Section}

Materials. $\mathrm{Os}(\mathrm{DPP})_{3}$ and $\mathrm{Ru}(\mathrm{DPP})_{3}$ were synthesized according to a literature procedure. ${ }^{29}$ They were purified by silica column chromatography with $2: 1 \mathrm{v} / \mathrm{v}$ acetonitrile/toluene. House-distilled $\mathrm{H}_{2} \mathrm{O}$ was passed through a four-cartridge Barnstead Nanopure II purification train consisting of Macropure pretreatment, Organics Free for removing trace organics, two ion exchangers, and a $0.2 \mathrm{~mm}$ hollow-fiber final filter for removing particles. Its resistivity was $18.3 \mathrm{M} \Omega \mathrm{cm}$. Octadecyltrichlorosilane(OTS) and (3-mercaptopropyl)trimethoxysilane (MPS) were from Petrarch Systems Inc. OTS was vacuumdistilled into sealed glass ampules, which were opened as needed immediately prior to the individual experiments. Octadecylmercaptan (OM) (Tokyo Kasei, Tokyo, Japan) and octadecanol (Aldrich) were used without further purification. Reagent grade $70 \% \mathrm{HClO}_{4}$ (Aldrich), chloroform (Fisher, ACS certified spectranalyzed), methanol (Fisher, spectroscopic grade), and all the other reagents were used as received.

Monolayer Techniques. The experiments at the air/water interface were carried out either in a KSV model 2200 Langmuir trough $\left(45 \times 15 \mathrm{~cm}^{2}\right)$ or in a smaller $\left(10 \times 20 \mathrm{~cm}^{2}\right)$ Teflon home-built trough. Each trough was equipped with a surface pressure Wilhelmy plate microbalance. Filter paper was used as the Wilhelmy plate. The morphology of the monolayer films at the air/water was investigated under controlled surface pressure conditions by Brewster angle microscopy (BAM 1 or Mini-BAM, Nanofilm Technologie GmbH, Göttingen, Germany). All experiments involving Langmuir troughs were done in inert gas enclosures. A more detailed protocol of Langmuir experiments has been described previously. ${ }^{26}$

X-ray Diffraction. Grazing incidence X-ray diffraction ${ }^{24,25}$ experiments were carried out in a thermostated, fully enclosed Langmuir trough placed on a liquid-surface diffractometer on the undulator beam line BW1 at HASYLAB, DESY in Hamburg, Germany. To avoid interference from low frequency convective surface waves, the water depth was made shallow $(<1 \mathrm{~mm})$ in the area under the X-ray footprint by inserting a glass block. A monochromatic X-ray synchrotron beam with a wavelength of $\lambda=1.481 \AA$ strikes the air/water interface at an incidence angle which is adjusted to be $0.85 \alpha_{c}\left(\alpha_{c} \cong 0.14^{\circ}\right.$ is a critical angle for total external reflection), illuminating a footprint of ca. $5 \times 40 \mathrm{~mm}^{2}$. The diffracted radiation passes through a Soller collimator that provides a resolution of $0.09^{\circ}$ in the in-plane scattering angle $2 \theta_{x y}$. The diffracted X-ray intensity is then detected by a linear position sensitive detector (OED-100-M, Braun, Garching, Germany) as a function of the vertical scattering angle $\alpha_{\mathrm{f}}$. The scattering of X-rays is elastic, and therefore the wave vectors $\boldsymbol{k}_{\mathrm{i}}$ and $\boldsymbol{k}_{\mathrm{f}}$ of the incident and diffracted photons have the same absolute value. The horizontal (in-plane) component of the scattering vector $\mathbf{Q}=\boldsymbol{k}_{\mathrm{i}}-\boldsymbol{k}_{\mathrm{f}}$ is given by

$$
\begin{array}{r}
Q_{x y}=\frac{2 \pi}{\lambda}\left(\cos ^{2} \alpha_{\mathrm{i}}+\cos ^{2} \alpha_{\mathrm{f}}-2 \cos \alpha_{\mathrm{i}} \cos \alpha_{\mathrm{f}} \cos 2 \theta_{x y}\right)^{2} \cong \\
\frac{4 \pi}{\lambda}\left(\sin \frac{2 \theta}{2}\right)
\end{array}
$$

and the vertical (out-of-plane) component by the following equation: ${ }^{24,25}$

$$
Q_{z}=\frac{2 \pi}{\lambda}\left(\sin \alpha_{\mathrm{i}}+\sin \alpha_{\mathrm{f}}\right) \cong \frac{2 \pi}{\lambda} \sin \alpha_{\mathrm{f}}
$$

Fabrication of Line Microband Electrodes. Electrochemical measurements at the air/water interface required specially designed "line" microelectrodes that can be positioned in the plane of the air/water interface. Details of their fabrication procedure have been described previously. ${ }^{26}$ Briefly, these electrodes are produced by creating a sharp gradient of wettability along a fracture line of ca. $800 \AA$ thick gold films vapordeposited $^{30}$ on microscope glass slides $\left(\mathrm{ca} .8 \times 20 \mathrm{~mm}^{2}\right.$ ). The pattern of the deposited gold film includes two circular areas, later used as electrical contact pads, and a strip of gold ( 0.4 to $6 \mathrm{~mm}$ in width) running between them. Following gold vapor deposition, monolayers of octadecane mercaptan and octadecyltrichlorosilane are formed on gold and on glass surfaces, respectively, by self-assembly. This renders all the surfaces of the substrate hydrophobic. By breaking such an electrode substrate in half (along a line drawn with a diamond pencil on the reverse side of the glass substrate perpendicular to the gold strip), one exposes a clean, and thus hydrophilic, edge surface of glass and gold and creates two identical microelectrodes. The newly exposed edge of gold forms a microband electrode 800 $\AA$ in width. The length of the microband is determined by the width of the vapor-deposited gold strip. It was varied from 0.4 to $6 \mathrm{~mm}$. Microelectrodes are positioned at the air/water interface by touching the water surface with the clean, hydrophilic edge of the electrode substrate. Thus, a line of wettability is formed along the edge of the gold micro-band between the hydrophilic gold cross-sectional area and the hydrophobic (OMcoated) front face of the gold strip. This line of wettability defines the "line" microelectrode contacting molecules spread at the air/water interface. The electrodes were always positioned at the air/water interface following monolayer spreading and solvent evaporation. At the air/water interface their performance is reproducible for ca. 30 to $60 \mathrm{~min}$. Subsequent slow contamination of the microband leads to a decrease of measured currents, and the electrode must be discarded.

Electrochemical Experiments. Cyclic voltammetry was performed with an Ensman Model 852 bipotentiostat (Bloomington, IN) in a three-electrode configuration under computer control. The reference electrode (SCE or a quasireference, Ag wire) and the $\mathrm{Pt}$ counter electrode were immersed in the subphase in a Langmuir trough behind the barrier where their presence did not interfere with a monolayer compression.

\section{Results and Discussion}

Monolayer Spreading and Compression. The structural formula of Os(DPP $)_{3}$ is shown in Figure 1 together with its surface pressure - area $(\pi-\mathrm{A})$ isotherm recorded on a $0.050 \mathrm{M}$ $\mathrm{HClO}_{4}$ subphase. As demonstrated earlier, while $\mathrm{Os}(\mathrm{DPP})_{3}$ is not an amphiphilic molecule, its chloroform solutions can be spread on a perchloric acid subphase where it forms stable monolayer films. ${ }^{22}$ Brewster angle microscopy revealed that, immediately upon spreading (mean molecular area of $360 \AA^{2}$ / 


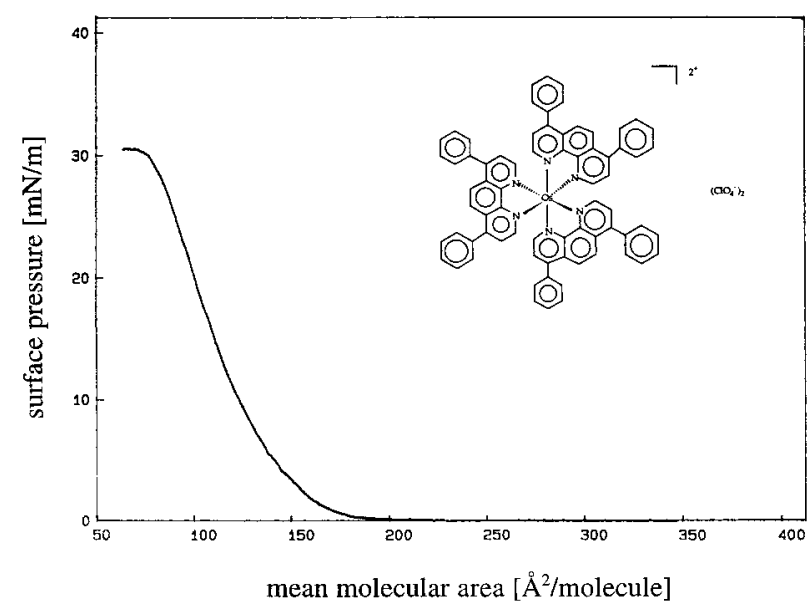

Figure 1. Pressure vs mean molecular area isotherm of Os(DPP $)_{3}$ recorded on $0.050 \mathrm{M} \mathrm{HClO}_{4}$ aqueous solution at $20{ }^{\circ} \mathrm{C}$. Os(DPP $)_{3}$ was spread from a $0.20 \mathrm{mM}$ chloroform solution at $360 \AA^{2} /$ molecule.
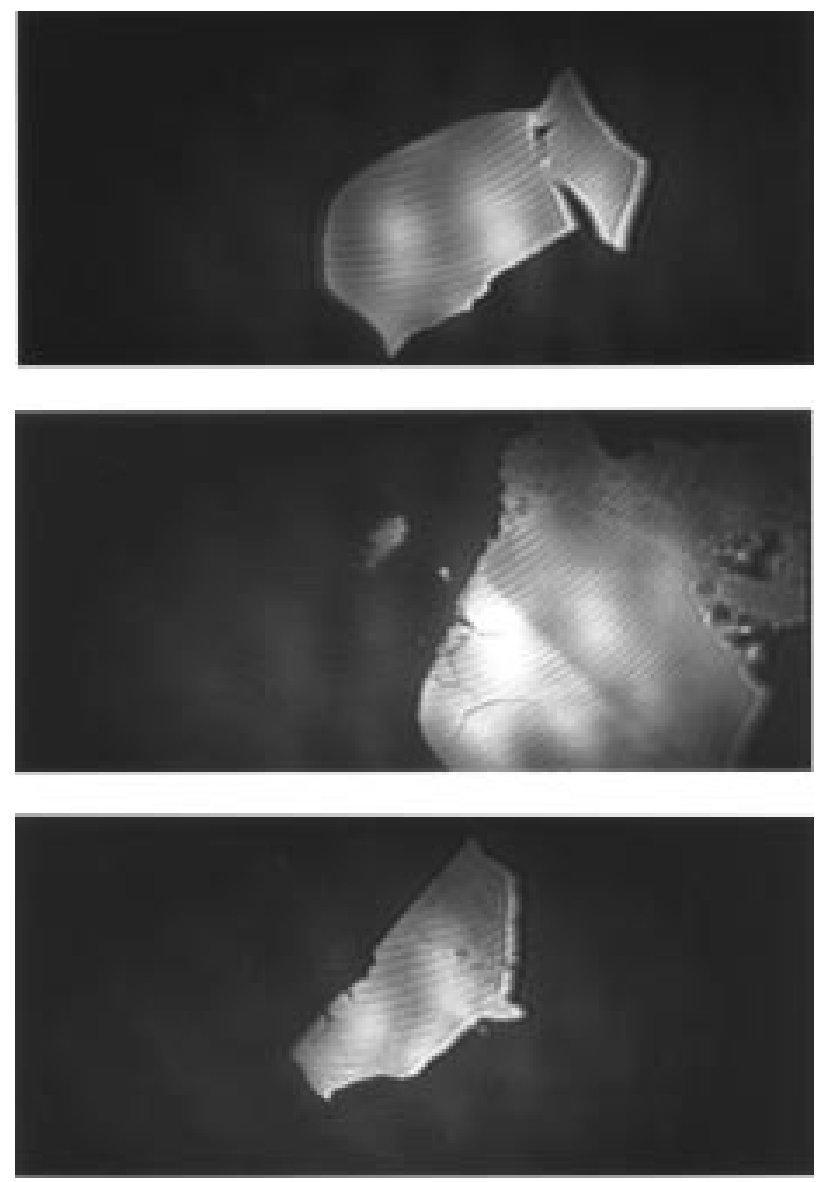

Figure 2. A selection of three BAM images of Os(DPP $)_{3}$ aggregates formed on the water surface $\left(0.050 \mathrm{M} \mathrm{HClO}_{4}\right.$ solution $)$ upon spreading at $360 \AA^{2} /$ molecule. The length of the shorter edge of the images corresponds to $730 \mu \mathrm{m}$.

molecule), Os(DPP $)_{3}$ forms solid 2D aggregates. The average size of these aggregates depends on the concentration of the chloroform spreading solutions. Relatively large aggregates (100-1000 $\mu \mathrm{m}$ diameter), such as those shown in Figure 2, are formed by spreading a $0.20 \mathrm{mM}$ Os(DPP $)_{3}$ solution. Decreasing the concentration of the spreading solution below ca. $0.1 \mathrm{mM}$ results in substantially smaller aggregates (ca. 1-10 $\mu \mathrm{m}$ diameter). Since we observed earlier that the kinetics of the lateral electron hopping measured in the compressed Os$(\mathrm{DPP})_{3}$ monolayers decreases significantly with the size of the

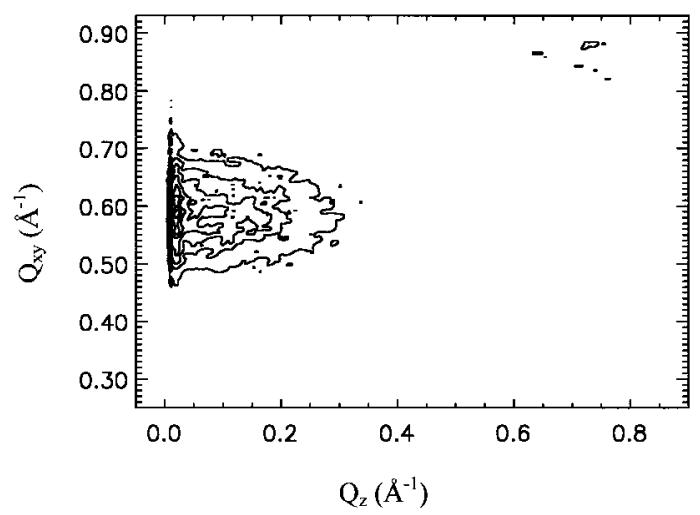

Figure 3. A contour plot of background-corrected X-ray intensities as a function of the in-plane and out-of-plane scattering vector components, $\mathrm{Q}_{x y}$ and $\mathrm{Q}_{z}$ obtained for $\mathrm{Os}(\mathrm{DPP})_{3}$ monolayer at $90 \AA^{2} /$ molecule at $20^{\circ} \mathrm{C}$. The monolayer spreading conditions were the same as those in Figure 1.

initially formed aggregates, ${ }^{22}$ all monolayer films were spread from $0.20 \mathrm{mM}$ chloroform solutions.

In view of these properties of the $\mathrm{Os}(\mathrm{DPP})_{3}$ monolayers, it is clear that the $\pi-\mathrm{A}$ isotherm shown in Figure 1 corresponds to the compression of the solid aggregates. Judging from our initial Brewster angle microscopioc investigations, this process leads ultimately to a gradual coalescence of the $2 \mathrm{D}$ aggregates and formation of largely homogeneous monolayer films at surface pressures of $25-30 \mathrm{mN} / \mathrm{m}$. This suggests that the aggregates are at least partially deformable. The full extent of compression, determined approximately from the large scale BAM images, results in a mean molecular area (MMA) of 90 $\pm 5 \AA^{2} /$ molecule. However, a higher magnification BAM image recorded during the final stages of compression revealed a somewhat heterogeneous texture of the monolayer. At $90 \pm 5$ $\AA^{2} /$ molecule, these images feature not only homogeneous areas but also areas of partial collapse. If, despite these inhomogeneities, a simplifying model is adopted that assumes an ideally 2D monolayer with a hexagonally close-packed structure of Os(DPP $)_{3}$, the MMA of $90 \AA^{2} /$ molecule corresponds to an average Os-Os distance of $10.2 \AA( \pm 6 \%)$.

Structure of the Os(DPP $)_{3}$ Monolayers at the Air/Water Interface. Grazing incidence angle X-ray diffraction is a wellestablished technique for structure elucidation of Langmuir monolayer films of a broad range of materials. ${ }^{24,25}$ The structure of Os(DPP $)_{3}$ monolayers was determined on a $0.050 \mathrm{M} \mathrm{HClO}_{4}$ subphase. The experiments were done at 205, 130, and $90 \AA^{2} /$ molecule. Not surprisingly, in view of the solid aggregate morphology of these monolayers, while the intensities increased progressively with compression, the diffraction patterns obtained in all of these cases were identical. Figure 3 presents a contour plot of X-ray intensities (corrected by background subtraction) as a function of the in-plane and out-of-plane scattering vector components, $\mathrm{Q}_{x y}$ and $\mathrm{Q}_{z}$, obtained at $90 \AA^{2} /$ molecule. As shown also in Figure 4 , the presence of a single maximum of the $\mathrm{Q}_{x y}$ component and the position of the maximum intensity of the $\mathrm{Q}_{z}$ vector at $0 \AA^{-1}$ indicate that $\mathrm{Os}(\mathrm{DPP})_{3}$ forms a hexagonal lattice in the plane of the air/water interface. ${ }^{24,25}$ The fact that the $\mathrm{Q}_{x y}$ intensity profiles are rather broad (fwhm is in a range of $\left.0.19-0.24 \AA^{-1}\right)$ suggests that positional coherence length is rather small (on the order of the lattice spacing). From the peak position of the in-plane component, $\mathrm{Q}_{x y}^{\max }$, one can obtain the lattice spacing $d_{h k}=2 \pi / Q_{x y}^{\max }=10.89 \AA$ and the Os-Os distance of $12.57 \AA$. The latter is $23 \%$ larger than its average value of $10.2 \AA$ calculated from the MMA corresponding, according to BAM (and electrochemical data presented below), 

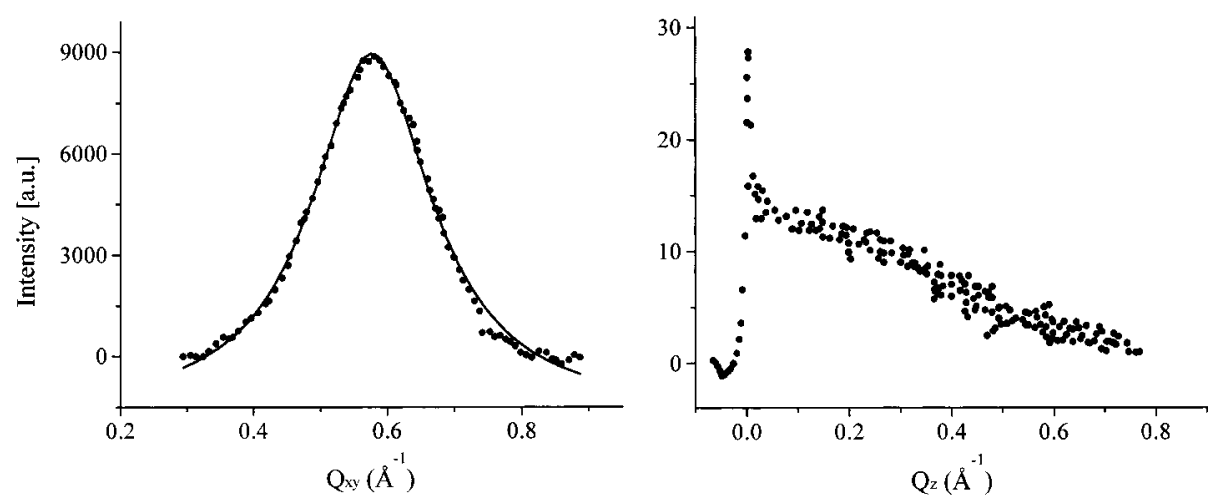

Figure 4. Diffraction intensities vs in-plane wave vector, $\mathrm{Q}_{x y}$, and the out-of-plane vector, $\mathrm{Q}_{z}$, recorded for Os(DPP) $)_{3}$ monolayer at $90 \AA^{2} / \mathrm{molecule}$ at $20{ }^{\circ} \mathrm{C}$.

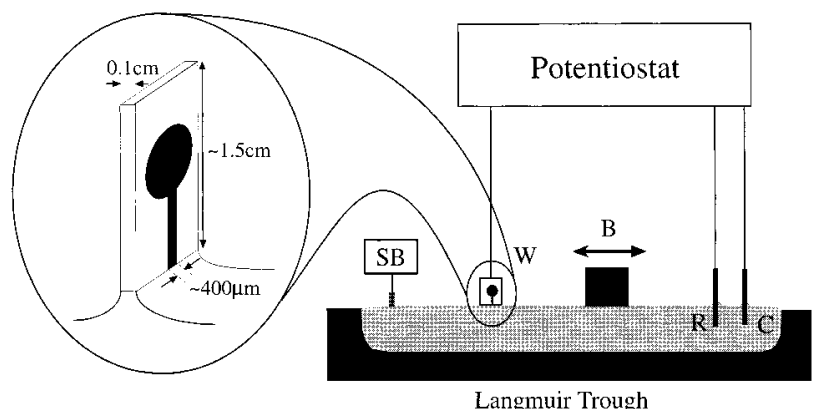

Figure 5. A schematic diagram of an experimental setup used to record preassure - MMA isotherms and 2D electrochemical data. Here B is the movable barrier of the Langmuir trough; $\mathrm{C}$ is the counter electrode; $\mathrm{R}$ is the reference electrode; $\mathrm{W}$ is the working electrode; $\mathrm{SB}$ is the surface film balance. The inset shows a line microelectrode as it touches the water surface.

to the conditions of full compression. Clearly, "full compression", judged on the basis of $\pi-\mathrm{A}$ isotherms and an initial appearance of a homogeneous texture in BAM images, corresponds already to a partially collapsed monolayer. Thus, Os(DPP $)_{3}$ cannot be compressed to form perfectly homogeneous 2D monolayers. The initially formed 2D solid aggregates, despite the homogeneous appearance of the BAM images near $90 \AA^{2} /$ molecule, cannot coalesce smoothly without forming some areas of collapsed structures.

It is interesting to compare the $2 \mathrm{D}$ crystal structure of Os$(\mathrm{DPP})_{3}$ with the $3 \mathrm{D}$ structure of $\mathrm{Ru}(\mathrm{DPP})_{3}$ described in the literature. ${ }^{31} \mathrm{We}$ assume that $\mathrm{Os}(\mathrm{DPP})_{3}$ and $\mathrm{Ru}(\mathrm{DPP})_{3}$ are isostructural, just as are a number of other bidentate octahedral Os and Ru complexes. ${ }^{32-35}$ In $3 \mathrm{D}, \mathrm{Ru}(\mathrm{DPP})_{3}$ chloride crystallizes in a monoclinic space group $P 2_{1 / c}$ with the cell dimensions $a=13.085 \AA, b=24.173 \AA$ and $c=22.773 \AA$, and $\beta=$ $110.91^{\circ} .31$ The distances between nearest $\mathrm{Ru}-\mathrm{Ru}$ neighbors range from 13.085 to $16.576 \AA$. Thus, the $2 \mathrm{D}$ crystal structure features a higher symmetry unit cell and shorter (12.57 $\AA$ ) metal-metal spacing.

Kinetics of the Lateral Electron Hopping. The electrochemical experiments employing a usual three electrode potentiostatic setup were carried out directly in a Langmuir trough. The key element in these experiments is a "line" microelectrode touching the water surface (see the schematic diagram of the experimental setup in Figure 5). The gold microelectrodes are fabricated by fractioning a small glass slide coated with an 800 $\AA$ thick vapor-deposited $\mathrm{Au}$ film that was initially pretreated with a self-assembled monolayer of octadecanethiol (see Experimental Section). The latter renders it hydrophobic. Since the clean surface of the cross-sectional area of the gold deposit exposed during fracture is hydrophilic, it remains in the subphase

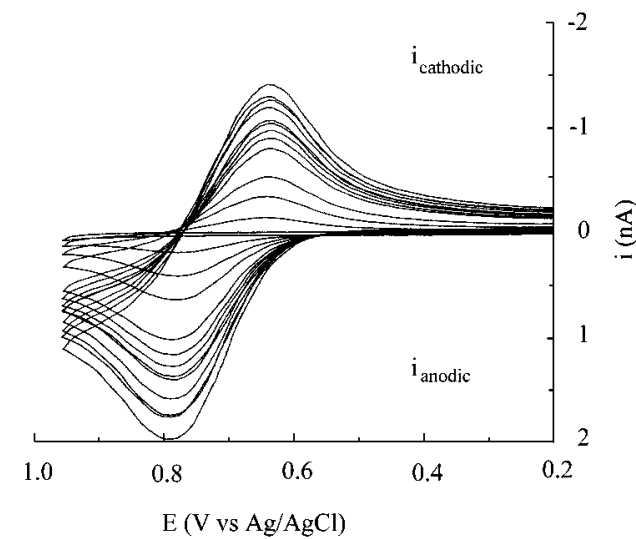

Figure 6. A set of $2 \mathrm{D}$ cyclic voltammograms of $\mathrm{Os}(\mathrm{DPP})_{3}$ monolayer at the air/water interface recorded concurrently with the monolayer compression. The CVs were recorded with a single $400 \mu \mathrm{m}$ line microelectrode at a scan rate of $0.2 \mathrm{~V} / \mathrm{s}$. Increasing current corresponds to decreasing mean molecular area of $\mathrm{Os}(\mathrm{DPP})_{3}$ from ca. 120 to 90 $\AA^{2} /$ molecule.

when the electrode substrate is brought in contact with its surface. Thus the line of wettability of the substrate touching the water surface (running between the clean cross-section and the octadecanethiol-coated gold surface) defines the line electrode. Also, wetting of the microband positions it precisely in the plane of the air/water interface where it contacts the Os$(\mathrm{DPP})_{3}$ monolayer.

Following monolayer spreading and incubation at $360 \AA^{2 /}$ molecule, a line electrode is positioned at the air/water interface. Subsequently, a series of cyclic voltammetric experiments is carried out while the monolayer is slowly compressed. Initially, only charging current is recorded since none of the 2D Os$(\mathrm{DPP})_{3}$ aggregates are in contact with the line electrode. ${ }^{36}$ The onset of the monolayer electrochemical activity corresponds approximately to a pressure of ca. $5 \mathrm{mN} / \mathrm{m}$ and a mean molecular area (MMA) of ca. $120 \AA^{2} /$ molecule. Subsequently, voltammetric current increases rapidly with the compression as shown in Figure 6 and reaches a maximum current at ca. 90 $\AA^{2} /$ molecule. Further compression does not initially result in changes of the peak current. Ultimately, however, (at MMA < $75 \AA^{2} /$ molecule) the voltammograms become ill-defined and irreproducible as a result of the monolayer collapse at the line electrode.

At any stage of compression resulting in a measurable faradaic current, the voltammetric curves exhibit characteristics of a reversible, diffusion-controlled process: the peak current is linearly dependent on $v^{1 / 2}$ ( $v$ is the scan rate) and the anodic and cathodic peak currents are equal in magnitude. ${ }^{26}$ The one exception is ca. $150-180 \mathrm{mV}$ peak-to-peak separation, a value 

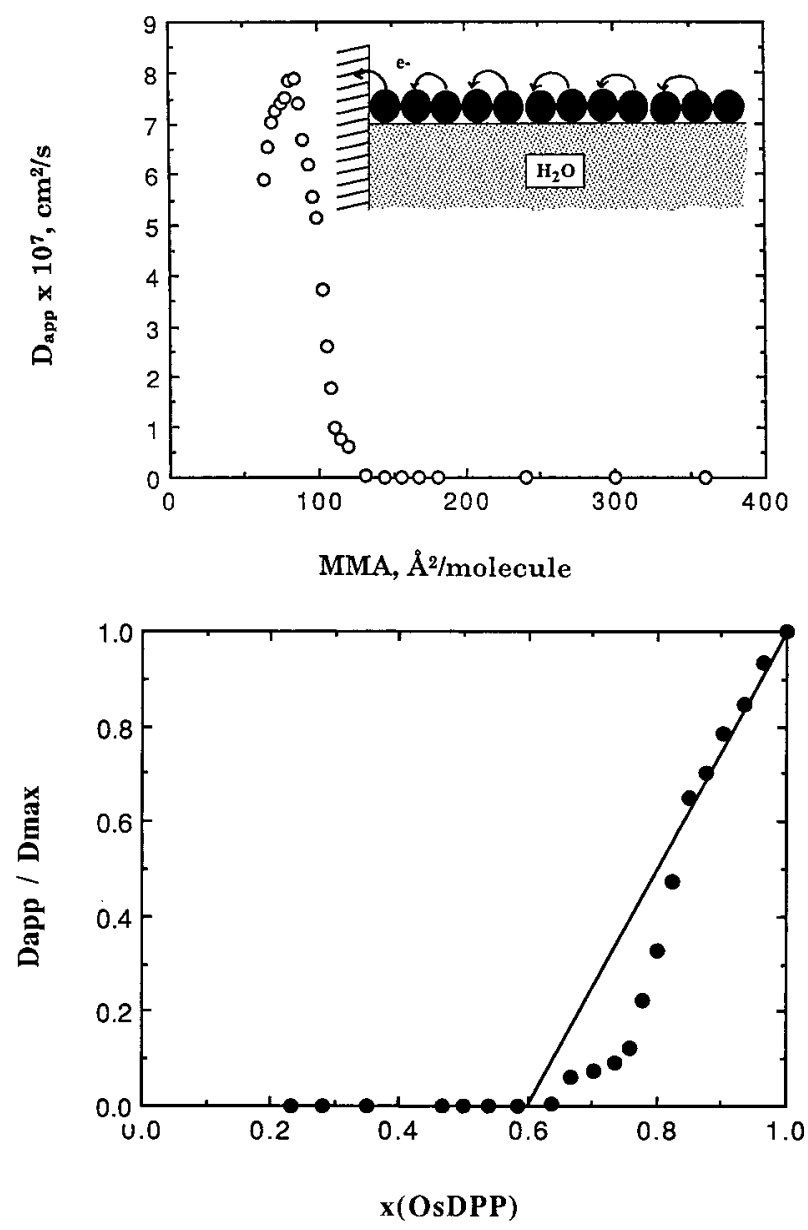

Figure 7. (A) A plot of $D_{\text {app }}$ vs mean molecular area obtained from the analysis of the anodic peak current data such as those in Figure 6. As shown schematically in the inset, $D_{\text {app }}$ characterizes the lateral electron hopping in Os(DPP $)_{3}$ monolayer. (B) The corresponding plot of $D_{\text {app }} / D_{\max }$ vs $X\left(\mathrm{Os}(\mathrm{DPP})_{3}\right)$. The line in part B reflects the prediction of the percolation theory.

substantially larger than ca. $60 \mathrm{mV}$ expected for a one electron oxidation/reduction process of $\mathrm{Os}(\mathrm{DPP})_{3}$ cycled between its $2+1$ $3+$ states. Since it does not depend on the scan rate (in the range 20 to $1000 \mathrm{mV} / \mathrm{s}$ ) it cannot be a result of either kinetic quasireversibility of the heterogeneous electron transfer or $i R$ loss due to uncompensated resistance. This large value is likely related to a variation in redox potential of the neighboring sites as a result of their proximity in the monolayer film ${ }^{37}$ and will be a subject of a separate report.

In view of the solid domain texture of these Os(DPP) monolayers, it is clear that the observed voltammetric current reflects diffusive lateral electron hopping within the 2D aggregates of the osmium centers (see inset in Figure 7A). The presence of the $2 \mathrm{D}$ aggregates eliminates the possibility of lateral diffusion of the individual Os(DPP $)_{3}$ molecules as a mechanism of charge transport responsible for the observed current. ${ }^{38}$ Each electron transfer event must be associated with a concurrent anion association or dissociation to accommodate electroneutrality of the system and the alternating $3+/ 2+$ oxidation state of $\mathrm{Os}(\mathrm{DPP})_{3}$. The access of perchlorate ions to the monolayer from the $50 \mathrm{mM}$ subphase is apparently unrestricted. The expression for a diffusion-controlled voltammetric peak current used in this $2 \mathrm{D}$ case $^{27}$

$$
i_{\mathrm{p}}=2.69 \times 10^{5} n^{2 / 3} l \Gamma^{*} D_{\mathrm{app}}^{1 / 2} v^{1 / 2}
$$

involves a product of $l \Gamma^{*}$, the length of the line electrode and the surface concentration of $\operatorname{Os}(\mathrm{DPP})_{3}\left(D_{\mathrm{app}}\right.$ is the apparent diffusion coefficient of the lateral electron hopping). The product reflects the reduced dimensionality of the system while its units are the same as those of $A C^{*}$, the electrode surface area and concentration used to describe the usual $3 \mathrm{D}$ voltammetric experiments. Using eq 3 to interpret the voltammetric data in Figure 6, one obtains the plot of $D_{\text {app }}$ vs average MMA shown in Figure 7A. The maximum value of $8.0 \times 10^{-7}( \pm 15 \%) \mathrm{cm}^{2} / \mathrm{s}$ is obtained at ca. $90 \AA^{2} /$ molecule, an MMA coinciding with the observation of monolayer coalescence in the low magnification BAM experiments.

Electron Hopping Percolation. Considering the fact that the Os-Os distances within aggregates are constant, independent of the monolayer compression, the increase of $D_{\text {app }}$ with compression in Figure 7A reflects an increasing extent of the monolayer electrochemical activity due to increasing connectivity between the individual 2D Os(DPP $)_{3}$ aggregates and between the aggregates and the line electrode. Accordingly, the data in Figure 7A should be viewed in terms of the percolation theory. ${ }^{39-41}$ To accomplish this, we replot (see Figure 7B) the $D_{\text {app }}$ vs MMA data as the normalized diffusion constant $D_{\text {app }} /$ $D_{\max }$ vs $X$, the fractional surface concentration of $\mathrm{Os}(\mathrm{DPP})_{3}$ at the air/water interface

$$
X=\Gamma / \Gamma_{\max }
$$

where $\Gamma_{\max }$ is the surface concentration corresponding to the maximum voltammetric current in Figure 7A. We assume that at that point all of the $\mathrm{Os}(\mathrm{DPP})_{3}$ sites are electrochemically active. Thus, $X$ is equivalent to "fractional loading", a percolation parameter equal to the ratio of the number of molecules in the lattice to the total number of lattice sites. However, expressing the loading in terms of fractional surface concentrations does not require selection of a particular lattice geometry. The continuous line in Figure $7 \mathrm{~B}$ represents predictions of the percolation theory for this $2 \mathrm{D}$ case. ${ }^{39}$ The source of the negative deviation appears to result from the fact that the percolation process is probed in this experiment with a $400 \mu \mathrm{m}$ long line electrode, an object comparable in size or smaller than the size of the 2D aggregates (see Figure 2). It is not surprising therefore that the current and the $D_{\text {app }} / D_{\max }$ in Figure $7 \mathrm{~B}$ increase, exhibiting an on-off type behavior rather than true percolation. If this interpretation is correct, increasing the length of the line electrodes should afford more equitable conditions of probing electron hopping percolation in this system. Figure 8 shows the results of such experiments where the normalized diffusion constant is plotted as a function of $X$ for a number of monolayer compression experiments involving line electrodes $0.4,1.0$, and $6.0 \mathrm{~mm}$ in length. It is readily apparent that when the hopping dynamics is averaged over sufficiently large areas of this 2D system by using line electrodes with lengths that are large compared to the average domain size, the system's behavior reflects predictions of $2 \mathrm{D}$ percolation theory. It is important to note that the meaningful increase of the surface area of our monolayer system probed electrochemically can be accomplished only by increasing the length of the microelectrodes. The average depth of electron hopping within the monolayer extending from the line electrode, or the diffusion layer thickness $(d)$ is, in comparison, very small. It can be assessed as $\delta \cong(D t)^{1 / 2}$ or ca. $10-20 \mu \mathrm{m}$.

Interpretation of the maximum value of the diffusion constant (Figure 7A) is complicated by the partially collapsed state of the monolayer discussed above. Analysis of the BAM, GIXD, and electrochemical data suggests that overcompression of Os$(\mathrm{DPP})_{3}$ monolayers is necessary to achieve the full extent of 
(a)

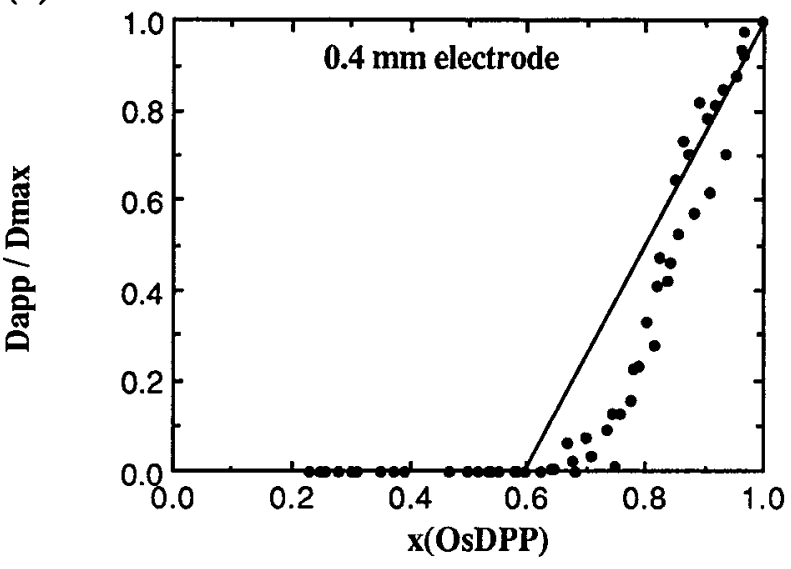

(b)

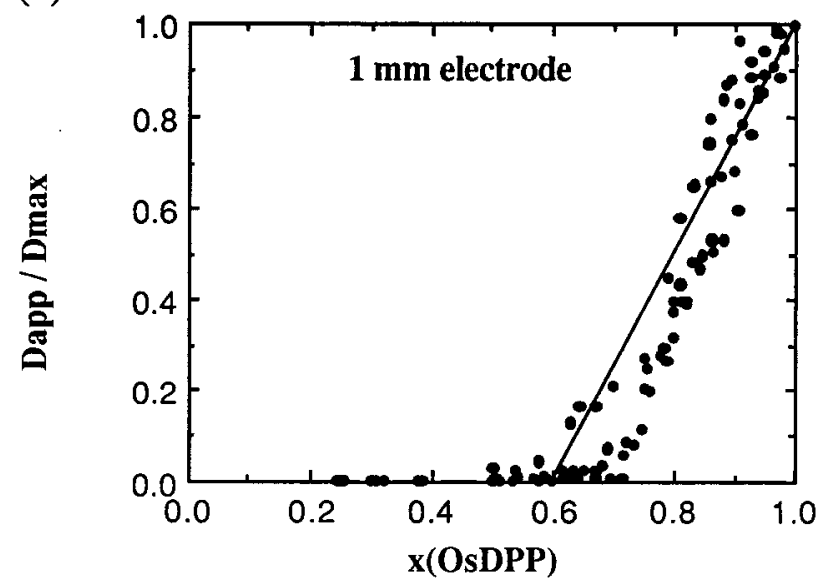

(c)

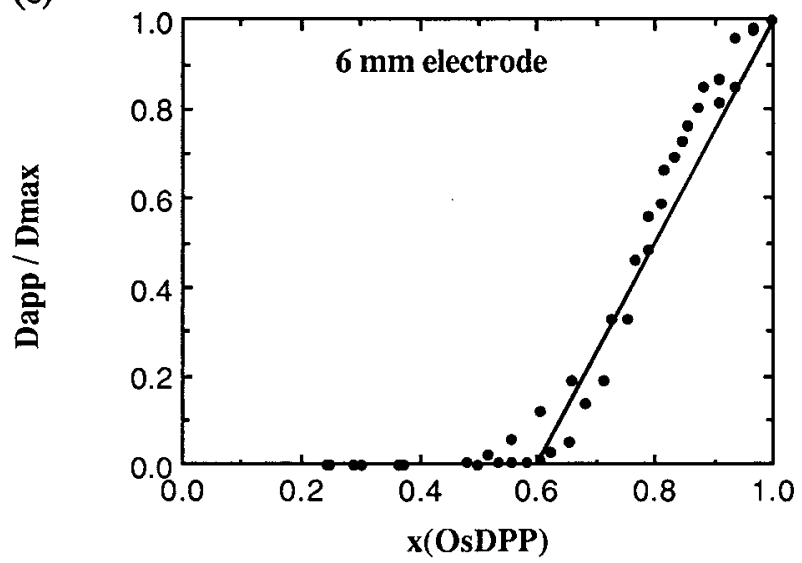

Figure 8. $D_{\text {app }} / D_{\max }$ vs $X\left(\mathrm{Os}(\mathrm{DPP})_{3}\right)$ plots obtained on the basis of several series of cyclic voltammetric experiments as those in Figure 6 recorded with $0.4,1.0$, and $6.0 \mathrm{~mm}$ long line microelectrodes.

electrochemical activity. Overcompression may be required to coalesce the aggregates and to achieve their full contact with the line electrode. Each of these may result in their partial collapse. Indeed, comparison of the apparent MMA at full compression determined by BAM of $90 \AA^{2} /$ molecule and the area per molecule within 2D aggregates obtained from GIXD data of $137 \AA^{2} /$ molecule indicates that the monolayer is partially collapsed at $90 \AA^{2} /$ molecule. What is not certain at this point is whether the collapsed regions are electroactive and contribute to the observed current flow. We may speculate that these regions are not electrochemically active since it is likely that access of the counterions is limited to only those aggregates that are in contact with the aqueous subphase. This would suggest that the effective concentration of $\mathrm{Os}(\mathrm{DPP})_{3}$ is smaller than that corresponding to the MMA of $90 \AA^{2} /$ molecule $(1.21$ $\times 10^{-10}$ vs $\left.1.84 \times 10^{-10} \mathrm{~mol} / \mathrm{cm}^{2}\right)$. Consequently, the maximum value of $D_{\text {app }}$ (Figure 7A) calculated from eq 3 has to be corrected and yields $D_{\text {app,cor }}=1.8 \times 10^{-6} \mathrm{~cm}^{2} / \mathrm{s}$.

In view of this discussion, it seems obvious that the lateral electron hopping process taking place in the $2 \mathrm{D}$ aggregates involves hexagonally packed Os(DPP $)_{3}$ centers spaced by $r=$ 12.6 $\AA$ (obtained from GIXD, and not $10.2 \AA$, an average value from the macroscopic BAM and electrochemical data). The maximum value of $D_{\text {app,cor }}$ can be interpreted in terms of the unimolecular rate constant $\left(k_{1}\right)$ of electron transfer between adjacent $\mathrm{Os}(\mathrm{DPP})_{3}$ molecules $^{40}$

$$
D=\frac{1}{4} k_{1} r^{2}
$$

This gives $k_{1}=4.7 \times 10^{8} \mathrm{~s}^{-1}$. To obtain the corresponding bimolecular rate constant of electron exchange $\left(k_{\mathrm{ex}}\right)$, we rely on the precursor complex model in which $k_{\mathrm{ex}}$ is expressed as a product of $k_{1}$ and $K_{\mathrm{p}}$, a precursor complex "equilibrium constant" 26,42

$$
k_{\mathrm{ex}}=k_{1} K_{\mathrm{p}} \quad K_{\mathrm{p}}=4 \pi N R^{2} \delta R
$$

Here, $\mathrm{N}$ is Avogadro number, $R=12.6$ is the radius of the excluded volume encompassing both reactants or an intermolecular distance between the reactants in the transition state, and $\delta R$ (ca. $0.5 \AA$ ) is the "reaction zone thickness". In view of eq $6, k_{\mathrm{ex}}=2.8 \times 10^{8} \mathrm{M}^{-1} \mathrm{~s}^{-1}$. This value is somewhat higher than the literature value of $7 \times 10^{7} \mathrm{M}^{-1} \mathrm{~s}^{-1}$ measured by NMR techniques in acetonitrile. ${ }^{43}$

A different way to probe electron hopping percolation is to examine the dependence of the electron hopping diffusion constant vs $\mathrm{X}\left(\mathrm{Os}(\mathrm{DPP})_{3}\right)$ in fully compressed, two-component monolayers. In such systems, addition of an inert component is intended to gradually increase the Os-Os distances. Dynamics of electron transport in 2D systems of randomly spaced sites have been treated theoretically by Blauch and Savéant. ${ }^{40,41}$ Besides percolation, distance dependence of electron transfer kinetics must be taken into account as the concentration of the diluent species is increased. Specifically a shift of the percolation threshold to lower densities of the electroactive species and higher values of the apparent diffusion coefficient have been predicted as a result of finite rates of electron transfer at distances larger than contact. ${ }^{41}$ Since one of our motivations has been to study long-range electron transfer kinetics, we attempted to form such two-component monolayers. Thus far, the results have been disappointing. $\mathrm{Ru}(\mathrm{DPP})_{3}$ is the only diluent species which we found to form homogeneous monolayer films with $\mathrm{Os}(\mathrm{DPP})_{3}$. This is not surprising since both compounds are isostructural. Several amphiphilic molecules were also used as potential diluents, but in all cases self-segregated, heterogeneous monolayers with $\mathrm{Os}(\mathrm{DPP})_{3}$ were obtained. The difference in lateral electron transport between a homogeneous $\mathrm{Os} / \mathrm{Ru}-$ $(\mathrm{DPP})_{3}$ and a heterogeneous Os(DPP $)_{3} /$ octadecanol monolayer is illustrated in Figure 9 where $D_{\text {app }} / D_{\max }$ vs $\mathrm{X}\left(\mathrm{Os}(\mathrm{DPP})_{3}\right)$ are plotted for the two cases. The data for the Os/Ru(DPP $)_{3}$ system follow essentially $2 \mathrm{D}$ percolation theory. A small shift of the percolation threshold to lower $X$ values could be the result of long-range electron transfer contributions in the electron hopping as described by Blauch and Savéant. ${ }^{41}$ However, limited precision of the experimental data does not allow us to make 


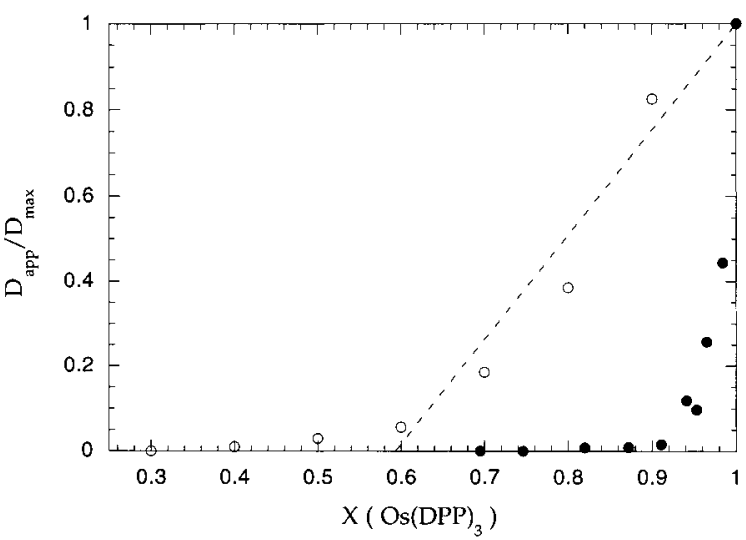

Figure 9. $D_{\mathrm{app}} / D_{\max }$ vs $X\left(\mathrm{Os}(\mathrm{DPP})_{3}\right)$ plots obtained for two-component monolayer films. The open circles correspond to Os(DPP $)_{3} / \mathrm{Ru}(\mathrm{DPP})_{3}$ and the closed circles to Os(DPP $)_{3} /$ octadecanol monolayers. All data were obtained with $400 \mu \mathrm{m}$ long line microelectrodes at ca. $25 \mathrm{mN} / \mathrm{m}$ with fully compressed films.

this statement with certainty. A substantial degree of selfsegregation of $\mathrm{Os}(\mathrm{DPP})_{3} /$ octadecanol results in a large shift of the percolation threshold to higher $X$ values.

\section{Conclusions}

Two dimensional electrochemical measurements carried out with line microelectrodes allow direct measurements of the kinetics of lateral electron hopping in Langmuir monolayers at the air/water interface. Percolation of electron hopping was observed in single component $\mathrm{Os}(\mathrm{DPP})_{3}$ monolayers during compression of their $2 \mathrm{D}$ solid aggregates reflecting an increasing extent of aggregate-aggregate and aggregate-microelectrode connectivity. Percolation behavior was also observed in homogeneous two-component $\mathrm{Os}(\mathrm{DPP})_{3} / \mathrm{Ru}(\mathrm{DPP})_{3}$ monolayers in which the latter component plays the role of a diluent of the osmium sites involved in the electron hopping. The electrochemically measured diffusion constants of electron hopping can be interpreted in terms of a unimolecular rate constant of the individual electron transfer processes between neighboring Os(DPP $)_{3}$ centers: $k_{1}=4.7 \times 10^{8} \mathrm{~s}^{-1}$. Thus, this technique can be used in systematic investigations of the kinetics of electron transfer processes involving redox species in Langmuir monolayers where the effects of molecular orientation, intermolecular distances, and solvent environment can be controlled.

Acknowledgment. This research was supported financially by the U.S. National Science Foundation under Grant CHE9422619. We thank Dr. David N. Blauch for his interest and helpful discussions at the early stages of this research.

\section{References and Notes}

(1) Kropf, M.; van Loyen, D.; Schwarz, O.; Durr, H. J. Phys. Chem. A 1998, 102, 5499-5505.

(2) Kropf, M.; Joselevich, E.; Durr, H.; Willner, I. J. Am. Chem. Soc. 1996, 118, 655-665.

(3) Sessler, J. L.; Wang, B.; Springer, S. L.; Brown, C. T. Electronand Energy-Transfer Reactions in Noncovalently Linked Supramolecular Model Systems: In Comprehensive Supramolecular Chemistry; Murakami, Y., Ed.; Elesevier Science Inc.: New York, 1996; Vol. 4, pp 311-336.

(4) Wiederrecht, G. P.; Niemczyk, M. P.; Svec, W. A.; Wasielewski, M. R. J. Am. Chem. Soc. 1996, 118, 81-88.

(5) Kuhn, H.; Möbius, D. Monolayer Assemblies: In Investigations of Surfaces and Interfaces; Rossiter, B. W., Baetzold, R. C., Eds.; John Wiley \& Sons: New York, 1993; Vol. IXB, Chapter 6, pp 375-542.

(6) Closs, G. L.; Miller, J. R. Science 1988, 240, 440-447.
(7) Boxer, S. G. Annu. Rev. Biophys. Biophys. Chem. 1990, 19, $267-$ 299.

(8) Kuki, A. Electronic Tunneling Paths in Proteins: In Structure and Bonding. Long-Range Electron Transfer in Biology; Springer-Verlag: Berlin, 1991; Vol. 75, pp 49-84

(9) Beratan, D. N.; Onuchic, J. N.; Winkler, J. R.; Gray, H. B. Science 1992, 258, 1740-1741.

(10) Isied, S. S.; Ogawa, M. Y.; Wishart, J. F. Chem. Rev. 1992, 92, 381-394.

(11) McLendon, G.; Hake, R. Chem. Rev. 1992, 92, 481-490.

(12) Moser, C. C.; Keske, J. M.; Warncke, K.; Farid, R. S.; Dutton, P. L. Nature 1992, 355, 796-802.

(13) Langen, R.; Chang, I.-J.; Germanas, J. P.; Richards, J. H.; Winkler, J. R.; Gray, H. B. Science 1995, 268, 1733-1735.

(14) Langen, R.; Colon, J. L.; Casimiro, D. R.; Karpishin, T. B.; Winkler,

J. R.; Gray, H. B. J. Biol. Inorg. Chem. 1996, 1, 221-225. 561.

(15) Gray, H. B.; Winkler, J. R. Annu. Rev. Biochem. 1996, 65, 537-

(16) Winkler, J. R.; Gray, H. B. J. Biol. Inorg. Chem. 1997, 2, 399404.

(17) Murray, R. W., Ed. Molecular Design of Electrode Surfaces; J. Wiley \& Sons: New York, 1992; Vol. 22.

(18) Bard, A. J. Integrated Chemical Systems. A Chemical Approach to Nonotechnology; J. Wiley \& Sons: New York, 1994

(19) Jortner, J.; Ratner, M., Eds. Molecular Electronics; IUPACBlackwell Science, Ltd.: Oxford, 1997.

(20) Terrill, R. H.; Murray, R. W. Electron Hopping Transport in Electrochemically Active, Molecular Mixed Valent Materials; in Molecular Electronics; Jortner, J., Ratner, M., Eds.; IUPAC-Blackwell Science, Ltd.: Oxford, 1997; pp 215-239.

(21) Majda, M. Dynamics of Electron Transport in Polymeric Assemblies of Redox Centers: In Molecular Design of Electrode Surfaces; Murray, R. W., Ed.; J. Wiley \& Sons: New York, 1992; pp 159-206.

(22) Charych, D. H.; Anvar, D. J.; Majda, M. Thin Solid Films 1994, $242,1-6$.

(23) Honig, D.; Möbius, D. J. Phys. Chem. 1991, 95, 4590-4592.

(24) Kjaer, K. Physica B 1994, 198, 100-109.

(25) Als-Nielsen, J.; Jackuemain, D.; Kjaer, K.; Leveiller, F.; Lahav, M.; Leizerowitz, L. Phys. Rep. 1994, 246, 251-313.

(26) Charych, D. H.; Landau, E. M.; Majda, M. J. Am. Chem. Soc. 1991 $113,3340-3346$

(27) Majda, M. Translational Diffusion and Electron Hopping in Monolayers at the Air/Water Interface: In Organic Thin Films and Surfaces: Ulman, A., Ed.; Academic Press: San Diego, 1995; Vol. 20, pp 331-347.

(28) Lee, W.-Y.; Hostetler, M. J.; Murray, R. W.; Majda, M. Isr. J. Chem. 1997, 37, 213-223.

(29) Gaudiello, J. G.; Bradley, P. G.; Norton, K. A.; Woodruff, W. H.; Bard, A. J. Inorg. Chem. 1984, 23, 3-10.

(30) Goss, C. A.; Charych, D. H.; Majda, M. Anal. Chem. 1991, 63, $83-88$

(31) Goldstein, B. M.; Barton, J. K.; Berman, H. M. Inorg. Chem. 1986, $25,842-847$

(32) Constable, E. C.; Raithby, P. R.; Smit, D. N. Polyhedron 1989, 8, 367-369.

(33) Richter, M. M.; Scott, B.; Brewer, K. J.; Willett, R. D. Acta Crystallogr. 1991, C47, 2443-2444.

(34) Biner, M.; Burgi, H.-B.; Rohr, C. J. Am. Chem. Soc. 1992, 114, $5197-5203$

(35) Krausz, E.; Riesen, H.; Rae, D. Aust. J. Chem. 1995, 48, 929.

(36) The entire cross-sectional area of the fractured gold-coated glass slide remains in contact with the subphase electrolyte. Thus the charging current is proportional to the surface area of the microband electrode of ca. $3 \times 10^{-7} \mathrm{~cm}^{2}$ for a typical $0.4 \mathrm{~mm}$ line electrode, interfacial capacitance (ca. $40 \mu \mathrm{F} / \mathrm{cm}^{2}$ ) and the rate of potential scan.

(37) Flanagan, J. B.; Margel, S.; Bard, A. J.; Anson, F. C. J. Am. Chem Soc. 1978, 100, 4248-4253.

(38) Our previous 2D voltammetric studies of liquid monolayer films of amphiphiles such as $\mathrm{N}$-alkanecarboxamide ferrocene derivatives at the air/water interface showed, as expected, decreasing voltammetric currents with monolayer compression reflecting decreasing lateral mobilities of these amphiphiles in monolayers of increasing population densities (see refs 26 , 27)

(39) Stauffer, D. Introduction to Percolation Theory; Taylor and Francis, Inc.: Philadelphia, 1985

(40) Blauch, D. N.; Savéant, J.-M. J. Am. Chem. Soc. 1992, 114, $3323-$ 3332.

(41) Blauch, D. N.; Savéant, J.-M. J. Phys. Chem. 1993, 97, 64446448.

(42) Hupp, J. T.; Weaver, M. J. J. Electroanal. Chem. 1983, 152, 1-14.

(43) Chan, M.-S.; Wahl, A. C. J. Phys. Chem. 1978, 82, 2542-2549. 